04.1

\title{
Метод конкурирующих реакций в исследовании процессов удаления паров ненасыщенных органических соединений в воздухе под действием плазмы импульсного разряда
}

\author{
(C) И.Е. Филатов, В.В. Уварин, Д.Л. Кузнецов
}

Институт электрофизики УрО РАН, Екатеринбург, Россия

E-mail: fil@iep.uran.ru

Поступило в Редакцию 11 июня 2019г.

В окончательной редакции 30 августа 2019г.

Принято к публикации 25 октября 2019г.

\begin{abstract}
Для исследования эффективности удаления паров летучих органических соединений из воздушных потоков при воздействии плазмы импульсных разрядов предлагается метод конкурирующих реакций. Используя в составе модельной смеси соединение с известными параметрами, можно оценивать аналогичные параметры других соединений с применением хроматографического метода анализа, не обладающего высоким временны́м разрешением. На примере паров стирола и метилметакрилата показано, что для ненасыщенных соединений их относительная реакционная способность в плазме разряда хорошо коррелирует с отношением констант скоростей их реакций с озоном.
\end{abstract}

Ключевые слова: коронный разряд, непредельные летучие органические соединения, метод конкурирующих реакций.

DOI: 10.21883/PJTF.2020.02.48954.17922

\begin{abstract}
Электрические разряды широко используются для очистки воздуха от паров летучих органических соединений (ЛОС) [1-3]. Исследование взаимодействия компонентов плазмы, генерируемой разрядами, с ЛОС связано с рядом проблем. Так, математическое моделирование процессов очистки воздуха затруднено из-за отсутствия данных о константах скоростей. Данные, полученные разными исследователями, трудно сравнивать из-за различных условий и больших погрешностей анализа. Ранее предложена методика, основанная на применении специальных модельных смесей [4,5], которая позволяет частично устранить эти недостатки. Используя смесь компонентов в равных условиях, можно сравнивать их реакционную способность и проводить относительные измерения с высокой точностью. Используя компонент с известными свойствами, можно оценить относительно него реакционные параметры других компонентов. Такой прием известен как метод конкурирующих реакций. В настоящей работе продемонстрированы возможности метода для исследования конверсии соединений в потоке воздуха в плазме импульсного разряда. В качестве объектов исследования выбраны ненасыщенные соединения: стирол и метилметакрилат (ММА), известные как мономеры для получения пластмасс. Двойная связь обеспечивает повышенную реакционную способность таких соединений по отношению к озону [6-8]. Известно, что кислород воздуха дезактивирует высокоактивный атомарный кислород, генерируемый разрядом, с образованием озона $\left(\mathrm{O}_{3}\right)$. Реакционная способность $\mathrm{O}_{3}$ недостаточна для обеспечения удаления большинства ЛОС. Для активации процессов окисления $\mathrm{O}_{3}$ in situ применяются комбинированные плазмокаталитические методы $[2,3]$, однако для исследования механизмов взаи-
\end{abstract}

модействия ЛОС с компонентами плазмы в газовой фазе лучше подходят некаталитические методы.

В эксперименте использовалась установка [4]. Параметры разряда были следующими: амплитуда импульса напряжения $120 \mathrm{kV}$, длительность $20 \mathrm{~ns}$, амплитуда тока 300 А. Энергия импульса $E_{p}$ вычислялась из осциллограмм $U(t)$ и $I(t)$ для каждой серии импульсов по формуле $E_{p}=\int U(t) I(t) d t$. Воздушная смесь обрабатывалась серией импульсов с частотой следования $f=10 \mathrm{~Hz}$. Длительность серии составляла $t=1 \mathrm{~min}$, пауза между сериями имела длительность $4 \mathrm{~min}$ и была необходима для выполнения анализа методом газожидкостной хроматографии. Удельная энергия $E$ вычислялась для серии импульсов по формуле $E=E_{p} f t / V$, где $V-$ объем газовой системы установки, $V=261$. Содержание $\mathrm{O}_{3}$ оценивалось по поглощению на длине волны $255 \mathrm{~nm}$ с помощью спектрофотометра „Specord-200“ с газовой кюветой толщиной $1 \mathrm{~cm}$ с использованием таблиц поглощения [9]. На рис. 1, $a$ показаны зависимости содержания стирола $\left(X_{1}\right)(1)$ и МMA $\left(X_{2}\right)(2)$ от удельной энергии $E$ и приведена оценка плазмохимического выхода озона $G_{O_{3}}$ в чистой воздушной смеси (3). Плазмохимические выходы $G[\mathrm{~mol} / 100 \mathrm{eV}]$ для удобства приведены на рис. $1, a$ с пересчетом размерностей: $1 \mathrm{ppm} \cdot 1 \cdot \mathrm{J}^{-1}=0.433 \mathrm{~mol} / 100 \mathrm{eV}$. Кривые 3.1 и 3.2 показывают наработку $\mathrm{O}_{3}$ в присутствии стирола и ММА соответственно. Стехиометрию реакции $\mathrm{O}_{3}$ можно оценить графически, как показано штриховыми линиями на рис. 1, $a$ : расчеты показывают, что одна молекула $\mathrm{O}_{3}$ участвует в удалении 0.86 и 0.92 молекул стирола и ММА соответственно. Очевидно, что $\mathrm{O}_{3}$ может расходоваться в неучтенных процессах, например при окислении побочных продуктов. Различие 

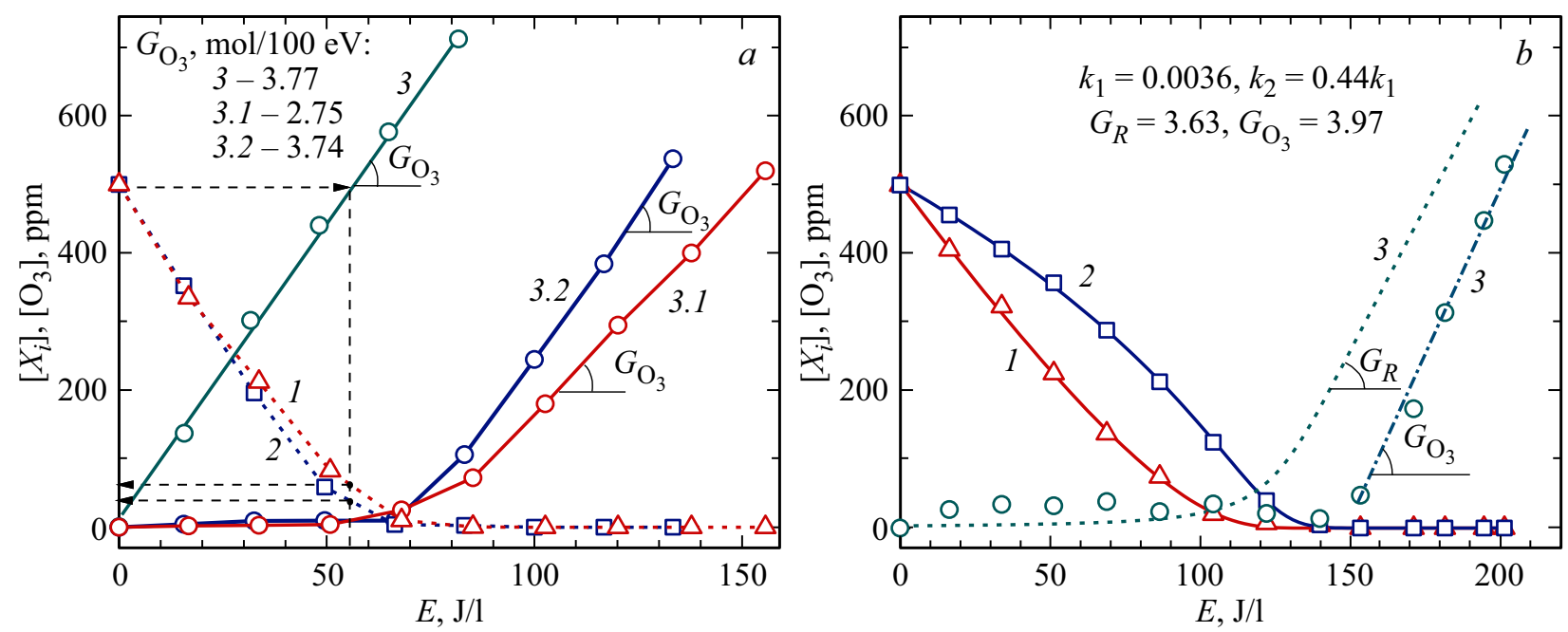

Рис. 1. $a-$ изменение концентраций $\left[X_{i}\right]$ и $\left[\mathrm{O}_{3}\right]$ в зависимости от удельной энергии $E$ в воздухе для стирола $(1)$, ММА $(2)$, озона в чистом воздухе (3), озона в присутствии стирола (3.1) и озона в присутствии ММА (3.2). $b-$ изменение концентраций $\left[X_{i}\right]$ и $\left[\mathrm{O}_{3}\right]$ в зависимости от удельной энергии $E$ в воздухе при одновременном присутствии стирола и ММА для стирола (1), MМА (2) и озона (3). Символы - эксперимент, линии - расчет.

наклона кривых 3.1 и 3.2 наработки $\mathrm{O}_{3}$ после удаления основного компонента также свидетельствует о его расходовании в дополнительных неучтенных процессах. Близость зависимостей 1 и 2 на рис. $1, a$ объясняется тем, что используемая методика анализа не позволяет оценивать реальную скорость взаимодействия компонентов: за время анализа успевают осуществиться и медленные процессы с характерными временами вплоть до десятков секунд. Это принципиальное ограничение „медленного“ метода анализа с помощью газожидкостной хроматографии. Учет этой особенности позволяет более точно интерпретировать результаты. Один из вариантов исследования „быстрых“ процессов с помощью „медленных“ методов анализа состоит в использовании смеси компонентов в равных условиях. Так, использование смеси паров стирола и ММА позволяет увидеть различие их реакционной способности в явном виде. На рис. $1, b$ символами показаны экспериментальные результаты. Для объяснения закономерностей удобно использовать формальный подход $[4,5,10]$. Концентрация примеси $\left[X_{i}\right]$ в зависимости от введенной в единицу объема газа удельной энергии $E$ упрощенно описывается системой уравнений

$$
\left\{\begin{array}{l}
\frac{d\left[X_{i}\right]}{d E}=-k_{i}\left[X_{i}\right][R], \\
\frac{d[R]}{d E}=G_{R}-\sum_{i=1}^{N} k_{i}\left[X_{i}\right][R],
\end{array}\right.
$$

где $[R]$ - концентрация формального реагента, $k_{i}$ - искомые константы, $i$ - номер компонента, $N$ - число компонентов, $G_{R}-$ плазмохимический выход реагента $R$. Для оценки величин констант $k_{i}$ можно использовать экспериментальные точки зависимостей $\left[X_{i}\right]$ от $E$, представленные на рис. $1, b$. Для решения обратной задачи системы уравнений (1) использовалось начальное приближение $G_{R} \approx \sum_{i=1}^{N} \Delta\left[X_{i}\right] / \Delta E$ при $E=0.1 E_{\max }$. С учетом закона действующих масс $k_{j} / k_{i} \approx\left[X_{i}\right] \Delta\left[X_{j}\right] /\left(\left[X_{j}\right] \Delta\left[X_{i}\right]\right)$. Вычисление безразмерной величины $k_{j} / k_{i}$ является основой метода конкурирующих реакций. В работе [8] данный метод используется для вычисления констант скоростей различных ненасыщенных соединений с озоном. Обратная задача для системы уравнений (1) при $N=2$ решалась в пакете MathCAD Prime 5.0 с аппроксимацией методом наименьших квадратов [11]. На рис. $1, b$ сплошными линиями показан результат моделирования, соответствующий найденным константам: $k_{1}=3.55 \cdot 10^{-3} 1 \cdot \mathrm{J} \cdot \mathrm{ppm}^{-1}$ и $k_{2}=1.56 \cdot 10^{-3} 1 \cdot \mathrm{J} \cdot \mathrm{ppm}^{-1}$ для стирола и ММА соответственно. Уточнено значение $G_{R}=3.63 \mathrm{~mol} / 100 \mathrm{eV}$. Из зависимостей следует, что основной частью формального реагента $R$ для данного класса соединений является $\mathrm{O}_{3}$. Смещение восходящей ветви кривой 3 для озона вправо указывает на то, что в нижней части уравнения (1) учтены не все процессы, ведущие к дополнительному расходованию $\mathrm{O}_{3}$. Важность измерения соотношения констант $k_{2} / k_{1}$, а не их абсолютных значений показана на рис. 2, $a$, где приведен фрагмент области данных, в которой концентрации $\left[X_{i}\right]$ и $[R]$ сравнимы. Так, увеличение абсолютных значений констант $k_{1}$ и $k_{2}$ при найденном при вычислении значении $k_{2} / k_{1}=0.44$ даже на два порядка при сохранении их соотношения практически не сказывается на зависимости для стирола и находится в пределах характерной погрешности анализа для ММА. Таким образом, используемый „медленный“ метод газожидкостной хроматографии дает возможность надежно определять соотношение констант $k_{2} / k_{1}$, однако не позволяет определять точные значения этих констант. На рис. 2, $b$ приведены экспериментальные 

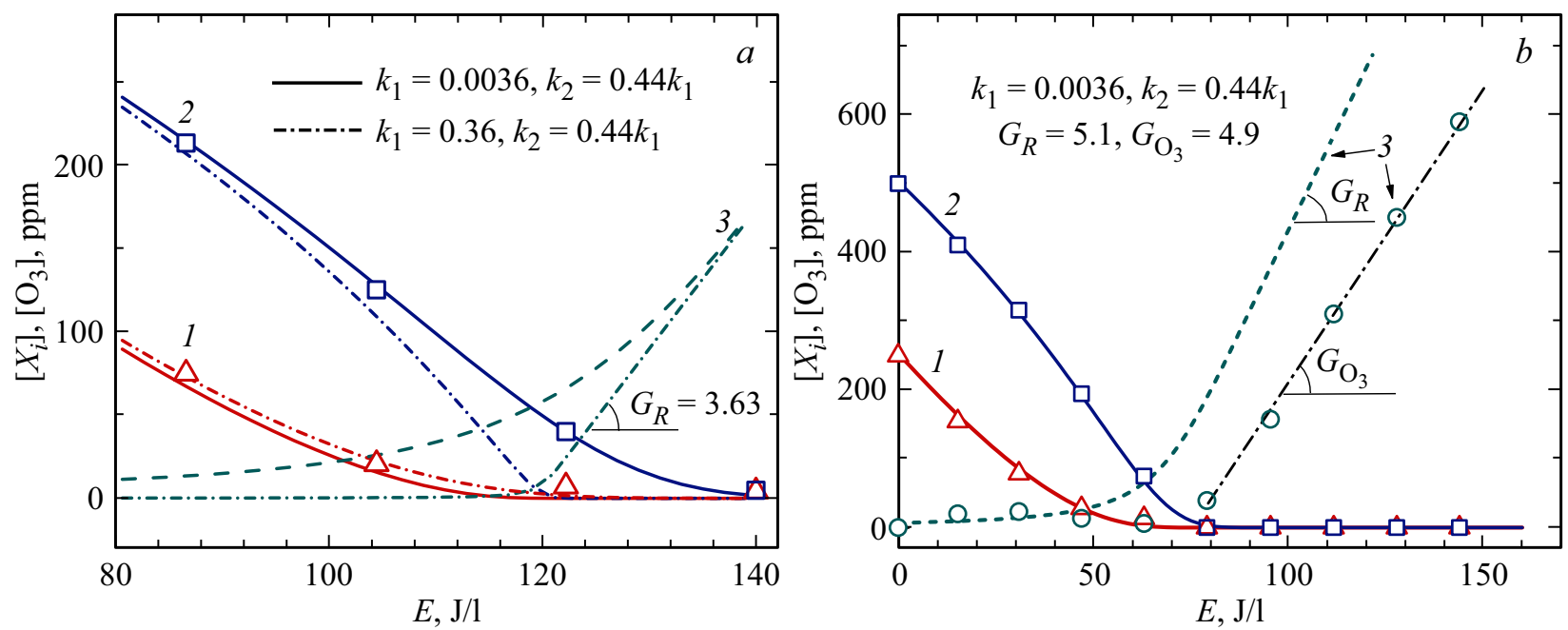

Рис. 2. $a-$ - фрагмент области данных, представленной на рис. $1, b$. Символы - эксперимент, сплошные линии - расчет, штрихпунктирные - расчет при увеличении констант $k_{1}$ и $k_{2}$ в 100 раз. $b$ - то же, что на рис. $1, b$, при других начальных концентрациях стирола и ММА.

данные для других начальных концентраций компонентов, а значение констант взято из решения обратной кинетической задачи (условия рис. $1, b)$, при этом наблюдается хорошее согласие эксперимента с вычисленными значениями. Отношение констант $k_{2} / k_{1}$ хорошо согласуется с отношением констант скоростей взаимодействия стирола $\left(k_{\mathrm{St}}\right)$ и ММА $\left(k_{\mathrm{MMA}}\right)$ с озоном: $k_{\mathrm{St}}=1.5 \cdot 10^{-17} \mathrm{~cm}^{3} /(\mathrm{mol} \cdot \mathrm{s}) \quad[12]$, $k_{\mathrm{MMA}}=6.7 \cdot 10^{-18} \mathrm{~cm}^{3} /(\mathrm{mol} \cdot \mathrm{s}) \quad$ [7]. При этом $k_{\mathrm{MMA}} / k_{\mathrm{St}}=0.45$, что очень близко к значению $k_{2} / k_{1}=0.44$. С использованием констант $k_{\mathrm{St}}$ и $k_{\mathrm{MMA}}$ решалась прямая кинетическая задача с учетом специфики измерения ( $1 \mathrm{~min}-$ работа, $4 \mathrm{~min}$ - пауза). Если принять $R \equiv \mathrm{O}_{3}$ и $G_{R} \equiv G_{\mathrm{O}_{3}}$, исходя из условий, представленных на рис. $1, b$, можно решить кинетическое уравнение, аналогичное системе уравнений (1). На рис. 3 линиями показаны расчетные данные, символами обозначены экспериментальные точки, аналогичные условиям, приведенным на рис. $1, b$. Видно, как меняется концентрация компонентов во время рабочего цикла и во время паузы. Характерные времена реакции $\mathrm{O}_{3}$ в области сравнимых концентраций $X_{i}$ и $\mathrm{O}_{3}$ достигают нескольких десятков секунд, и проявляется общий второй порядок процесса, в то время как в области начальных концентраций процесс удаления примеси хорошо описывается прямой (нулевой порядок).

Таким образом, показано, что основным действующим агентом при удалении ненасыщенных соединений с помощью импульсного разряда в воздухе является озон. Применяемый метод конкурирующих реакций позволяет с высокой точностью оценивать реакционную способность органического соединения по отношению к компонентам плазмы, если это соединение используется в смеси с другим соединением, параметры для которого известны. Этот метод позволит получать необходимые параметры для значительного количества ЛОС без ис-

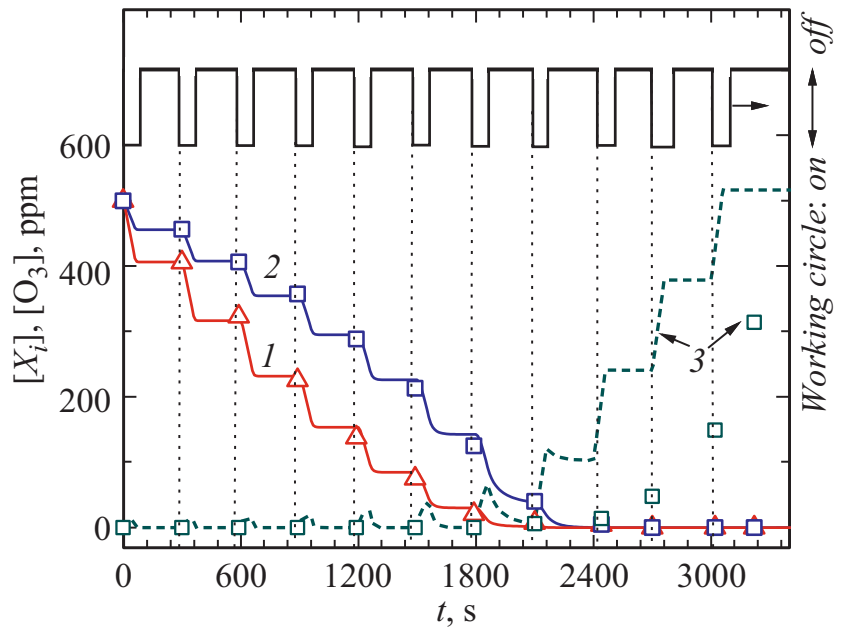

Рис. 3. Изменение концентраций $\left[X_{i}\right]$ и $\left[\mathrm{O}_{3}\right]$ в зависимости от времени $t$ в воздухе для стирола (1), MМА (2) и озона (3) с учетом специфики измерения (1 min - работа, $4 \mathrm{~min}-$ пауза, как показано на диаграмме вверху, правая оси ординат). Символы - эксперимент, линии - кинетические расчеты.

пользования дорогостоящей техники исследования быстрых процессов.

\section{Финансирование работы}

Работа выполнена при финансовой поддержке Российского фонда фундаментальных исследований (проект № 17-08-01212a).

\section{Конфликт интересов}

Авторы заявляют, что у них нет конфликта интересов. 


\section{Список литературы}

[1] Vandenbroucke A.M., Morent R., De Geyter N., Leys C. // J. Hazard. Mater. 2011. V. 195. P. 30-54.

DOI: 10.1016/J.JHAZMAT.2011.08.060

[2] Chung W.C., Mei D.H., Tu X., Chang M.B. // Catal. Rev. 2019. V. 61 . N 2. P. $270-331$.

DOI: $10.1080 / 01614940.2018 .1541814$

[3] Plasma chemistry and catalysis in gases and liquids / Eds P. Lukes, M. Magureanu, V.I. Pârvulescu. Wiley-VCH, 2012. $401 \mathrm{p}$.

[4] Филатов И.Е., Уварин В.В., Кузнецов Д.Л. // ЖТФ. 2018. T. 88. B. 5. C. $702-710$.

DOI: 10.21883/JTF.2018.05.45898.2421

[5] Filatov I.E., Uvarin V.V., Kuznetsov D.L. // J. Phys.: Conf. Ser. 2019. V. 1147. N 1. P. 012122.

DOI: $10.1088 / 1742-6596 / 1147 / 1 / 012122$

[6] Poznyak T.I., Oria I.C., Poznyak A.S. Ozonation in the gaseous phase // Ozonation and biodegradation in environmental engineering. Elsevier, 2019. P. 325-349. DOI: 10.1016/B978-0-12-812847-3.00021-4

[7] Le Person A., Eyglunent G., Daële V., Mellouki A., Mu Y. // J. Photochem. Photobiol. 2008. V. 195. N 1. P. 54-63. DOI: 10.1016/J.JPHOTOCHEM.2007.09.006

[8] Al Mulla I., Viera L., Morris R., Sidebottom H., Treacy J., Mellouki A. // ChemPhysChem. 2010. V. 11. N 18. P. 4069 4078.

DOI: $10.1002 / \mathrm{cphc} .201000404$

[9] Molina L.T., Molina M.J. // J. Geophys. Res.: Atmospheres. 1986. V. 91. N D13. P. 14501-14508.

DOI: $10.1029 / J D 091$ iD13p14501

[10] Yan K., Heesch E.J.M., Pemen A.J.M., Huijbrechts P.A.H.J. // Plasma Chem. Plasma Process. 2001. V. 21. N 1. P. 107-137. DOI: $10.1023 / \mathrm{A}: 1007045529652$

[11] Korobov V.I., Ochkov V.F. Chemical kinetics with Mathcad and Maple. Vienna: Springer, 2011. P. 115-156.

[12] Bernard F., Eyglunent G., Daele V., Mellouki A. // J. Phys. Chem. A. 2010. V. 114. N 32. P. 8376-8383.

DOI: $10.1021 / \mathrm{jp} 104451 \mathrm{v}$ 